\title{
GENERALIZED NEYMAN-PEARSON LEMMA VIA CONVEX DUALITY*
}

\author{
JAKŠA CVITANIĆ \\ Department of Mathematics \\ University of Southern California \\ Los Angeles, CA 90089 \\ cvitanic@math.usc.edu
}

\author{
IOANNIS KARATZAS \\ Departments of Mathematics and Statistics \\ Columbia University \\ New York, NY 10027 \\ ik@math.columbia.edu
}

June 2, 2007

\begin{abstract}
We extend the classical Neyman-Pearson theory for testing composite hypotheses versus composite alternatives, using a convex duality approach as in Witting (1985). Results of Aubin \& Ekeland (1984) from non-smooth convex analysis are employed, along with a theorem of Komlós (1967), in order to establish the existence of a max-min optimal test in considerable generality, and to investigate its properties. The theory is illustrated on representative examples involving Gaussian measures on Euclidean and Wiener space.
\end{abstract}

Key words: Hypothesis testing, optimal generalized tests, saddle-points, stochastic games, Komlós theorem, non-smooth convex analysis, subdifferentials, normal cones. AMS 1991 Subject Classifications: Primary 62C20, 62G10; secondary 49N15, 93E05. Running Title: Neyman-Pearson theory via Convex Duality.

${ }^{*}$ Research supported in part by the National Science Foundation, under Grant NSF-DMS-97-32810. 


\section{Introduction}

On a measurable space $(\Omega, \mathcal{F})$, suppose that we are given two probability measures $Q$ ("hypothesis") and $P$ ("alternative"), and that we want to discriminate between them. We can try to do this in terms of a (pure) test, that is, a random variable $X: \Omega \rightarrow\{0,1\}$, which rejects $Q$ on the event $\{X=1\}$. With this interpretation, $Q(X=1)$ is the probability of rejecting $Q$ when it is true (probability of type-I-error), whereas $P(X=0)=1-P(X=1)$ is the probability of accepting $Q$ when it is false (probability of type-II-error).

Ideally, one would like to minimize these error probabilities simultaneously, but typically this will not be possible: a more sensitive radar decreases the chance of letting enemy aircraft go undetected, but also makes false alarms more likely. The next best thing is then to fix a certain number $0<\alpha<1$ (say $\alpha=1 \%$ or $\alpha=5 \%$ ), and try to

$$
\text { maximize } P(X=1) \text {, subject to } Q(X=1) \leq \alpha \text {. }
$$

In other words, one tries to find a test that minimizes the probability of type-II-error, among all tests that keep the probability of type-I-error below a given acceptable significance level $\alpha \in(0,1)$. This is the tack taken by the classical Neyman-Pearson theory of hypothesis testing; see, for instance, Lehmann (1986), Ferguson (1967) or Witting (1985).

The basic results of this theory are very well known. Suppose that $\mu$ is a third probability measure with

$$
P \ll \mu, \quad Q \ll \mu
$$

(for instance, $\mu=(P+Q) / 2)$, and set

$$
G:=\frac{d P}{d \mu}, \quad H:=\frac{d Q}{d \mu} .
$$

Then the problem of (1.1) has a solution, namely

$$
\hat{X}=\mathbf{1}_{\{\hat{z} H<G\}},
$$

provided that

$$
Q(\hat{z} H<G)=\alpha \text { for some } 0<\hat{z}<\infty .
$$

In other words, the test $\hat{X}$ of (1.4) rejects the hypothesis, if and only if the "likelihood ratio" $G / H=(d P / d \mu) /(d Q / d \mu)$ is sufficiently large.

When a number $\hat{z}$ with the properties (1.5) cannot be found, one has to consider randomized tests, that is, random variables $X: \Omega \rightarrow[0,1]$. The new interpretation is that, if the outcome $\omega \in \Omega$ is observed, then the hypothesis $Q$ is rejected (respectively, accepted) with probability $X(\omega)$ (resp., $1-X(\omega)$ ), independently of everything else. This way,

$$
\mathbf{E}^{P}(X)=\int X(\omega) P(d \omega)
$$


is then the power of the randomized test $X$, that is, the probability of rejecting the hypothesis $Q$ when it is false; and

$$
\mathbf{E}^{Q}(X)=\int X(\omega) Q(d \omega)
$$

is the probability of type-I-error for the randomized test $X$ (i.e., of rejecting $Q$ when it is true). By analogy with (1.1), one seeks a randomized test $\hat{X}$ which

$$
\left\{\begin{array}{c}
\text { maximizes } \mathbf{E}^{P}(X), \text { over all randomized tests } \\
X: \Omega \rightarrow[0,1] \text { with } \mathbf{E}^{Q}(X) \leq \alpha
\end{array}\right\}
$$

The advantage of this "randomized" formulation is that the problem of (1.8) has a solution for any given significance level $\alpha \in(0,1)$. In particular, the supremum

$$
\sup _{X \in \mathcal{X}_{\alpha}} \mathbf{E}^{P}(X), \quad \text { with } \quad \mathcal{X}_{\alpha}:=\left\{X: \Omega \rightarrow[0,1] / \mathbf{E}^{Q}(X) \leq \alpha\right\},
$$

is attained by the randomized test

$$
\hat{X}=\mathbf{1}_{\{\hat{z} H<G\}}+b \cdot \mathbf{1}_{\{\hat{z} H=G\}}
$$

in $\mathcal{X}_{\alpha}$, where we have set (with the convention $0 / 0=0$ ):

$$
\begin{gathered}
\hat{z}:=\inf \{u \geq 0 / Q(u H<G) \leq \alpha\}, \\
b:=\frac{\alpha-Q(\hat{z} H<G)}{Q(\hat{z} H=G)}=\frac{\alpha-Q(\hat{z} H<G)}{Q(\hat{z} H \leq G)-Q(\hat{z} H<G)} \in[0,1] .
\end{gathered}
$$

\section{Composite hypotheses and alternatives}

Let us suppose now that, on the measurable space $(\Omega, \mathcal{F})$, we have an entire family $\mathbf{Q}$ of probability measures (composite "hypothesis"), which we want to discriminate against another family $\mathbf{P}$ of probability measures (composite "alternative"). By analogy with (1.2), we asssume

$$
\begin{gathered}
\mathbf{P} \cap \mathbf{Q}=\emptyset, \\
P \ll \mu, \quad Q \ll \mu ; \quad \forall P \in \mathbf{P}, Q \in \mathbf{Q}
\end{gathered}
$$

for some probability measure $\mu$, and set

$$
\begin{gathered}
G_{P}:=\frac{d P}{d \mu}(P \in \mathbf{P}), \quad H_{Q}:=\frac{d Q}{d \mu}(Q \in \mathbf{Q}) \\
\mathcal{X}_{\alpha}:=\left\{X: \Omega \rightarrow[0,1] / \mathbf{E}^{Q}(X) \leq \alpha, \forall Q \in \mathbf{Q}\right\}
\end{gathered}
$$


as in (1.3), (1.9). Since we have now an entire family $\mathbf{P}$ of alternatives, we shall replace (1.9) by the max-min criterion

$$
\underline{V}(\alpha):=\sup _{X \in \mathcal{X}_{\alpha}}\left(\inf _{P \in \mathbf{P}} \mathbf{E}^{P}(X)\right)
$$

In other words, we shall look for a randomized test $\hat{X}$ that maximizes the smallest power

$$
\gamma(X):=\inf _{P \in \mathbf{P}} \mathbf{E}^{P}(X)
$$

attainable through measures of the family $\mathbf{P}$, over all randomized tests $X$ of size

$$
s(X):=\sup _{Q \in \mathbf{Q}} \mathbf{E}^{Q}(X) \leq \alpha
$$

Definition 2.1 If such a randomized test $\hat{X} \in \mathcal{X}_{\alpha}$ exists, it will be called max-min-optimal for testing the (composite) hypothesis $\mathbf{Q}$ against the (composite) alternative $\mathbf{P}$, at the given level of significance $\alpha \in(0,1)$.

Under appropriate conditions on the family $\mathbf{P}$ of alternatives, we shall see in the next section that an optimal max-min randomized test exists and has a form reminiscent of (1.10), namely

$$
\hat{X}=\mathbf{1}_{\{\hat{z} \hat{H}<\hat{G}\}}+B \cdot \mathbf{1}_{\{\hat{z} \hat{H}=\hat{G}\}} .
$$

Here $B$ is a random variable with values in the interval $[0,1]$; the random variable $\hat{G}$ is of the form $G_{\hat{P}}=d \hat{P} / d \mu$ of (2.3) for some $\hat{P} \in \mathbf{P}$; the random variable $\hat{H}$ is chosen from a suitable family that contains the convex hull

$$
C o(H ; \mathbf{Q}):=\left\{\lambda H_{Q_{1}}+(1-\lambda) H_{Q_{2}} / Q_{1} \in \mathbf{Q}, Q_{2} \in \mathbf{Q}, 0 \leq \lambda \leq 1\right\}
$$

of $\left\{H_{Q}\right\}_{Q \in \mathbf{Q}}$; and $\hat{z}$ is a suitable positive number.

As we shall see, it is no longer possible to compute these quantities by means of formulae as explicit as (1.11) and (1.12), which are valid in the simple-hypothesis-vs.-simplealternative case. However, methods of nonsmooth convex analysis and duality theory provide both the existence of these quantities and algorithms that can lead to their computation, as illustrated by several examples in Section 5. The main result of the present paper, namely Theorem 4.1 (and its Corollaries 4.1 and 4.2), shows that the associated dual problem always (that is, even with uncountably many hypotheses and/or alternatives) has a solution; that there is never a duality gap; and that the optimal test always has the $0-1$ representation (2.8).

The idea of using convex duality methods in Hypothesis Testing is not new; it goes back to the paper by Krafft \& Witting (1967), and is developed to a considerable extent in Chapter 
2 of the treatise by Witting (1985), particularly Sätze 2.80 and 2.81 on pp. 267-274. See also the papers by Lehmann (1952), Baumann (1968) and Huber \& Strassen (1973), as well as pp. 361-362 in the book by Vajda (1989), for related results. The paper by Baumann (1968) proves the existence of the max-min-optimal test using general duality results from the theory of linear programming, as well as weak-compactness arguments. We provide a different, self-contained proof for existence, using an almost-sure convergence argument based on the theorem of Komlós (1967). In addition, our approach enables us to show that the optimal test is always of the Neyman-Pearson form (2.8), thereby giving a characterization potentially useful in finding algorithms for computing the optimal test. To the best of our knowledge, the characterization (2.8) has only been known under stronger conditions on the null and the alternative hypotheses. We obtain it here in a very general setting, using infinitedimensional nonsmooth convex optimization results of Aubin \& Ekeland (1984), apparently not used previously in the theory of hypothesis-testing. Our own inspiration came from Heath (1993), who used the Neyman-Pearson lemma as a tool for solving a stochastic control problem that can also be treated by methods of convex duality; for related work along this line see Karatzas (1997), as well as Cvitanić \& Karatzas (1999), Cvitanić (2000), Föllmer \& Leukert (1999, 2000) and Spivak (1998) for similar problems arising in the context of Mathematical Finance.

\section{Results: Analysis}

Let us begin the statement of results by introducing the set of random variables

$$
\mathcal{H}:=\left\{H \in \mathbf{L}^{1}(\mu) / H \geq 0, \mu-a . e . \text { and } \mathbf{E}^{\mu}(H X) \leq \alpha, \forall X \in \mathcal{X}_{\alpha}\right\}
$$

As is relatively straightforward to check (cf. Section 6), this set is convex, bounded in $\mathbf{L}^{1}(\mu)$, closed under $\mu$-a.e. convergence, and contains the convex hull of (2.9), namely

$$
C o(H ; \mathbf{Q}) \subseteq \mathcal{H}
$$

In a similar spirit, we shall impose the following assumption throughout.

Assumption 3.1 The set of densities

$$
\mathcal{G}:=\left\{G_{P}\right\}_{P \in \mathbf{P}}
$$

is convex and closed under $\mu$-a.e. convergence. (From (2.3), the convexity of $\mathcal{G}$ is equivalent to the convexity of the family $\mathbf{P}$ of alternatives.) 
The set $\mathcal{G}$ of $(3.3)$ is obviously bounded in $\mathbf{L}^{1}(\mu)$, since $\mathbf{E}^{\mu}\left(G_{P}\right)=P(\Omega)=1$ for every $P \in \mathbf{P}$. We shall comment in Remark 4.1 below, on the necessity of imposing Assumption 3.1 and of considering the class $\mathcal{H}$ of (3.1), as we do. Now the key observation is that, for arbitrary $G \in \mathcal{G}$ and $H \in \mathcal{H}$, we have $G=d P / d \mu$ for some $P \in \mathbf{P}$, and thus

$$
\begin{aligned}
\mathbf{E}^{P}(X) & =\mathbf{E}^{\mu}(G X)=\mathbf{E}^{\mu}[X(G-z H)]+z \cdot \mathbf{E}^{\mu}(H X) \\
& \leq \mathbf{E}^{\mu}(G-z H)^{+}+\alpha z ; \quad \forall z>0, \quad \forall X \in \mathcal{X}_{\alpha}
\end{aligned}
$$

from (3.1) and $0 \leq X \leq 1$ in (1.9). Furthermore, we have equality in (3.4) for some $\hat{G} \in \mathcal{G}$, $\hat{H} \in \mathcal{H}, \hat{z} \in(0, \infty)$ and $\hat{X} \in \mathcal{X}_{\alpha}$, if and only if the conditions

$$
\begin{gathered}
\mathbf{E}^{\mu}(\hat{H} \hat{X})=\alpha \\
\hat{X}=\mathbf{1}_{\{\hat{z} \hat{H}<\hat{G}\}}+B \cdot \mathbf{1}_{\{\hat{z} \hat{H}=\hat{G}\}}, \mu-\text { a.e. }
\end{gathered}
$$

both hold, for some random variable $B: \Omega \rightarrow[0,1]$; and in this case, with $\hat{P}:=\int \hat{G} d \mu \in \mathbf{P}$, we have

$$
\mathbf{E}^{\hat{P}}(\hat{X})=\mathbf{E}^{\mu}(\hat{G} \hat{X})=\alpha \hat{z}+\mathbf{E}^{\mu}(\hat{G}-\hat{z} \hat{H})^{+} .
$$

Proposition 3.1 Suppose that there exists a quadruple $(\hat{G}, \hat{H}, \hat{z}, \hat{X}) \in\left(\mathcal{G} \times \mathcal{H} \times(0, \infty) \times \mathcal{X}_{\alpha}\right)$ that satisfies (3.5), (2.8), and

$$
\mathbf{E}^{\mu}[\hat{X}(\hat{G}-G)] \leq 0, \quad \forall G \in \mathcal{G}
$$

Then we have

$$
\mathbf{E}^{\hat{P}}(X) \leq \mathbf{E}^{\hat{P}}(\hat{X}) \leq \mathbf{E}^{P}(\hat{X}) ; \quad \forall X \in \mathcal{X}_{\alpha}, P \in \mathbf{P} .
$$

In other words, the pair $(\hat{X}, \hat{P})$ with $\hat{P}=\int \hat{G} d \mu$, is then a saddle-point for the stochastic game with lower-value $\underline{V}(\alpha)$ as in (2.5), and upper-value

$$
\bar{V}(\alpha):=\inf _{P \in \mathbf{P}}\left(\sup _{X \in \mathcal{X}_{\alpha}} \mathbf{E}^{P}(X)\right),
$$

namely:

$$
\underline{V}(\alpha)=\bar{V}(\alpha)=\mathbf{E}^{\hat{P}}(\hat{X})=\int \hat{G} \hat{X} d \mu .
$$

We collect in Section 6 the proofs of all the results in the paper. The inequality of (3.4) also points the way to a duality approach, that will lead eventually to the existence of a quadruple $(\hat{G}, \hat{H}, \hat{z}, \hat{X})$ with the properties (3.5)-(3.7) and (2.8), as follows. Let us introduce the value function

$$
\tilde{V}(z) \equiv \tilde{V}(z ; \alpha):=\inf _{(G, H) \in(\mathcal{G} \times \mathcal{H})} \mathbf{E}^{\mu}(G-z H)^{+}, \quad 0<z<\infty
$$


of an auxiliary dual problem, and observe from (3.4) the inequality

$$
\bar{V}(\alpha) \leq V_{*}(\alpha)
$$

where we have set

$$
V_{*}(\alpha):=\inf _{\substack{z>0 \\(G, H) \in(\mathcal{G} \times \mathcal{H})}}\left[\alpha z+\mathbf{E}^{\mu}(G-z H)^{+}\right]=\inf _{z>0}[\alpha z+\tilde{V}(z)]
$$

Proposition 3.2 Under the assumptions of Proposition 3.1, the following hold:

(i) The pair $(\hat{G}, \hat{H})$ attains the infimum in (3.11) with $z=\hat{z}$.

(ii) The triple $(\hat{z}, \hat{G}, \hat{H})$ attains the first infimum in (3.13).

(iii) The number $\hat{z} \in(0, \infty)$ attains the second infimum in (3.13).

(iv) There is no "duality gap" in (3.12); namely, $V_{*}(\alpha)=\bar{V}(\alpha)=\underline{V}(\alpha)=\mathbf{E}^{\hat{P}}(\hat{X})$.

We shall show in the next section how to select the "dual variables" $\quad(\hat{z}, \hat{G}, \hat{H}) \in$ $((0, \infty) \times \mathcal{G} \times \mathcal{H})$ in such a way, that the optimal "primal variable" (generalized test) $\hat{X}$ is then given in the form (2.8).

\section{Results: Synthesis}

We can follow now the above reasoning in reverse, and try to obtain the existence of the quadruple $(\hat{G}, \hat{H}, \hat{z}, \hat{X})$ postulated in Proposition 3.1, by characterizing its constituent elements in terms of the properties of Proposition 3.2. This is done in Lemmata 4.1-4.4 and in Theorem 4.1 below, using nonsmooth convex analysis as our main tool; cf. Aubin \& Ekeland (1984), Chapters 1-4.

Lemma 4.1 The function $\tilde{V}(\cdot)$ of (3.11) is Lipschitz-continuous:

$$
\left|\tilde{V}\left(z_{1}\right)-\tilde{V}\left(z_{2}\right)\right| \leq\left|z_{1}-z_{2}\right|, \quad \forall 0<z_{1}, z_{2}<\infty
$$

Lemma 4.2 For any given $z \geq 0$, there exists a pair $(\hat{G}, \hat{H})=\left(\hat{G}_{z}, \hat{H}_{z}\right) \in \mathcal{G} \times \mathcal{H}$ that attains the infimum in (3.11).

Lemma 4.3 For any given $\alpha \in(0,1)$, there exists a number $\hat{z}=\hat{z}_{\alpha}>0$ that attains the infimum $V_{*}(\alpha)=\inf _{z>0}[\alpha z+\tilde{V}(z)]$ of $(3.13)$.

Lemma 4.4 Under the norm $\|(G, H, z)\|:=\mathbf{E}^{\mu}(|G|+|H|)+|z|$, the set

$$
\mathcal{M}:=\{(G, H, z) \in \mathcal{L} / G \in \mathcal{G}, H \in \mathcal{H}, z \geq 0\}
$$


is a closed, convex subset of $\mathcal{L}:=\mathbf{L}^{1}(\mu) \times \mathbf{L}^{1}(\mu) \times \Re$. Furthermore, the functional

$$
\mathcal{L} \ni(G, H, z) \longmapsto \tilde{U}(G, H, z):=\alpha z+\mathbf{E}^{\mu}(G-H)^{+} \in(0, \infty)
$$

is proper, convex and lower-semicontinuous on $\mathcal{L}$; and $(G, H, z) \mapsto \tilde{U}(G, z H, z)=\alpha z+$ $\mathbf{E}^{\mu}(G-z H)^{+}$attains its infimum over $\mathcal{M}$ at the triple $(\hat{G}, \hat{H}, \hat{z})$, with $\hat{z} \equiv \hat{z}_{\alpha}$ as in Lemma 4.3 and $(\hat{G}, \hat{H}) \equiv\left(\hat{G}_{\hat{z}}, \hat{H}_{\hat{z}}\right)$ as in Lemma 4.2.

Let us consider now the dual $\mathcal{L}^{*}:=\mathbf{L}^{\infty}(\mu) \times \mathbf{L}^{\infty}(\mu) \times \Re$ of the space $\mathcal{L}=\mathbf{L}^{1}(\mu) \times \mathbf{L}^{1}(\mu) \times \Re$, the set $\mathcal{M}_{*}:=\{(G, z H, z) /(G, H, z) \in \mathcal{M}\}$, the normal cone

$$
\begin{aligned}
N(\hat{G}, \hat{z} \hat{H}, \hat{z}):=\left\{(W, X, y) \in \mathcal{L}^{*} \quad\right. & \quad \mathbf{E}^{\mu}(\hat{G} W+\hat{z} \hat{H} X)+\hat{z} y \geq \\
& \left.\geq \quad \mathbf{E}^{\mu}(G W+z H X)+z y, \forall(G, H, z) \in \mathcal{M}\right\}
\end{aligned}
$$

to this set $\mathcal{M}_{*}$ at the point $(\hat{G}, \hat{z} \hat{H}, \hat{z}) \in \mathcal{M}_{*}$, and the subdifferential at this point

$$
\begin{aligned}
\partial \tilde{U}(\hat{G}, \hat{z} \hat{H}, \hat{z}) & :=\left\{(W, X, y) \in \mathcal{L}^{*} / \tilde{U}(\hat{G}, \hat{z} \hat{H}, \hat{z})-\tilde{U}(G, Y, z) \leq\right. \\
& \left.\leq \mathbf{E}^{\mu}[W(\hat{G}-G)+X(\hat{z} \hat{H}-Y)]+y(\hat{z}-z), \quad \forall(G, Y, z) \in \mathcal{L}\right\}
\end{aligned}
$$

of the functional $\tilde{U}$ in (4.3). From Lemma 4.4 and non-smooth convex analysis, as in Corollary 4.6.3 of Aubin \& Ekeland (1984), we know that

$$
(\hat{G}, \hat{z} \hat{H}, \hat{z}) \in \mathcal{M}_{*} \quad \text { is a solution of } \quad(0,0,0) \in \partial \tilde{U}(\hat{G}, \hat{z} \hat{H}, \hat{z})+N(\hat{G}, \hat{z} \hat{H}, \hat{z}) .
$$

In other words, there exists a triple $(\hat{W}, \hat{X}, \hat{y})$, such that $(\hat{W}, \hat{X}, \hat{y}) \in N(\hat{G}, \hat{z} \hat{H}, \hat{z})$ and $(-\hat{W},-\hat{X},-\hat{y}) \in \partial \tilde{U}(\hat{G}, \hat{z} \hat{H}, \hat{z})$, or equivalently

$$
\begin{gathered}
\mathbf{E}^{\mu}[\hat{W}(\hat{G}-G)]+\mathbf{E}^{\mu}[\hat{X}(\hat{z} \hat{H}-z H)]+\hat{y}(\hat{z}-z) \geq 0 ; \quad \forall(G, H, z) \in \mathcal{M} \\
\mathbf{E}^{\mu}\left[\hat{W}(G-\hat{G})+\hat{X}(Y-\hat{z} \hat{H})+(G-Y)^{+}-(\hat{G}-\hat{z} \hat{H})^{+}\right] \geq(\alpha+\hat{y})(\hat{z}-z) ; \quad \forall(G, Y, z) \in \mathcal{L} .
\end{gathered}
$$

Sending $z \rightarrow \pm \infty$, we observe that (4.7) can hold only if

$$
\hat{y}=-\alpha .
$$

Reading (4.6) with $\hat{y}=-\alpha$ and with $G=\hat{G}, H=\hat{H}, z=\hat{z} \pm \delta$ for $\delta>0$, we obtain

$$
\mathbf{E}^{\mu}(\hat{H} \hat{X})=\alpha
$$

as postulated by (3.5). On the other hand, reading (4.6) with $G=\hat{G}, z=\hat{z}$, we obtain

$$
\mathbf{E}^{\mu}(H \hat{X}) \leq \mathbf{E}^{\mu}(\hat{H} \hat{X})=\alpha, \quad \forall H \in \mathcal{H}
$$

in conjunction with (3.5)(a); and reading (4.6) with $H=\hat{H}, z=\hat{z}$ leads to

$$
\mathbf{E}^{\mu}[\hat{W}(\hat{G}-G)] \geq 0, \quad \forall G \in \mathcal{G} .
$$


Theorem 4.1 The random variable $\hat{X}$ belongs to $\mathcal{X}_{\alpha}$, and satisfies

$$
\hat{X}=-\hat{W}=\mathbf{1}_{\{\hat{z} \hat{H}<\hat{G}\}}+B \cdot \mathbf{1}_{\{\hat{z} \hat{H}=\hat{G}\}} \quad, \quad \mu-\text { a.e. }
$$

for a suitable random variable $B: \Omega \rightarrow[0,1]$.

Corollary 4.1 The stochastic game with lower-(resp., upper-) value $\underline{V}(\alpha)$ (resp., $\bar{V}(\alpha))$ as in (2.5), (3.9) has saddle-point $(\hat{X}, \hat{P})$ with $\hat{P}=\int \hat{G} d \mu$, and value $\underline{V}(\alpha)=\bar{V}(\alpha)=\mathbf{E}^{\hat{P}}(\hat{X})$.

This follows from Proposition 3.1, since the conditions (3.5) and (2.8) are satisfied (recall (4.9) and (3.5)(a)), and so is condition (3.7), because (3.7)' and (4.9) imply

$$
\mathbf{E}^{\mu}[\hat{X}(\hat{G}-G)]=\mathbf{E}^{\mu}[\hat{W}(G-\hat{G})] \leq 0, \quad \forall G \in \mathcal{G} .
$$

Corollary 4.2 The randomized test $\hat{X} \in \mathcal{X}_{\alpha}$ is max-min optimal for testing the composite hypothesis $\mathbf{Q}$ against the composite alternative $\mathbf{P}$ in the sense of Definition 2.1; namely, it maximizes the smallest value of the power $\gamma(X)=\inf _{P \in \mathbf{P}} \mathbf{E}^{P}(X)$ attainable over alternatives $P \in \mathbf{P}$, against all generalized tests $X \in \mathcal{X}_{\alpha}$ of size $s(X):=\sup _{Q \in \mathbf{Q}} \mathbf{E}^{Q}(X) \leq \alpha$.

Remark 4.1 If the convex set $\tilde{\mathcal{H}}:=C o(H ; \mathbf{Q})$ of $(2.9)$ is itself closed under $\mu-$ a.e. convergence, there is no need to introduce the larger set $\mathcal{H}$ of (3.1) and (3.2), since the auxiliary dual problem

$$
\tilde{V}(z):=\inf _{(G, H) \in(\mathcal{G} \times \tilde{H})} \mathbf{E}^{\mu}(G-z H)^{+}
$$

has then a solution in $\mathcal{G} \times \tilde{H}$.

Similarly, it is well-known that an optimal test can often be found among "Bayesian tests" (see Witting (1985), for example). More precisely, suppose that the set $\left\{H_{Q} / Q \in \mathbf{Q}\right\}$ can be represented in the form $\left\{H_{\theta} / \theta \in \Theta\right\}$ for some measurable space $\{\Theta, \mathcal{E}\}$, such that $(\theta, \omega) \mapsto H_{\theta}(\omega)$ is an $\mathcal{F} \otimes \mathcal{E}-$ measurable function on $\Omega \times \Theta$. Let $\mathcal{S}$ be a set of probability measures ("prior distributions") on $\{\Theta, \mathcal{E}\}$, and denote

$$
\mathcal{H}_{\mathcal{S}}:=\left\{\int H_{\theta} d S(\theta) / S \in \mathcal{S}\right\} .
$$

Again, if $\mathcal{H}_{\mathcal{S}}$ is convex and closed under $\mu$-a.e. convergence, and if $\left\{H_{Q} / Q \in \mathbf{Q}\right\} \subseteq \mathcal{H}_{\mathcal{S}}$, we can work with the set $\mathcal{H}_{\mathcal{S}}$ instead of $\mathcal{H}$.

However, we cannot relax the convexity and closedness Assumption 3.1 on the set $\mathcal{G}$ of (3.3); in particular, we cannot replace $\mathcal{G}$ by the larger, convex set

$$
\tilde{\mathcal{G}}:=\left\{G \in \mathbf{L}^{1}(\mu) / G \geq 0, \mu-\text { a.e.; } \mathbf{E}^{\mu}(G X) \geq \inf _{P \in \mathbf{P}} \mathbf{E}^{P}(X), \forall X \in \mathcal{X}_{\alpha}\right\} .
$$

One reason why this might not work is that $\tilde{\mathcal{G}}$ does not have to be closed under $\mu$-a.e. convergence, since Fatou's lemma can then fail to produce the desired inequality in $(3.3)^{\prime}$. 


\section{$5 \quad$ Examples}

We present in this section a few representative examples, involving hypothesis testing for Gaussian measures on Euclidean and Wiener spaces. Most, if not all, of these examples are probably well-known; they are developed here only insofar as they allow us to illustrate the theory of Sections 3 and 4 in a transparent and direct way.

Let us consider first the case of the filtered measurable space $\{\Omega, \mathcal{F}\}, \mathbf{F}:=\{\mathcal{F}(t)\}_{0 \leq t \leq 1}$, where $\Omega:=C\left([0,1] ; \Re^{d}\right)$ is the set of all continuous functions $\omega:[0,1] \rightarrow \Re^{d}, W(t, \omega):=\omega(t)$, $0 \leq t \leq 1$ is the coördinate mapping process, $\mathcal{F}(t):=\sigma(W(s) ; 0 \leq s \leq t)$, and $\mathcal{F}=\mathcal{F}(1)$. Consider also the space $\Theta$ of $\mathbf{F}$-progressively measurable processes $\theta:[0,1] \times \Omega \rightarrow K$ for some given compact, convex subset $K$ of $\Re^{d}$ with $0 \notin K$. We shall look at Wiener measure $\mu$ on $(\Omega, \mathcal{F})$, as well as at the family of measures $\left\{\mu_{\theta}, \theta \in \Theta\right\}$ given by

$$
\frac{d \mu_{\theta}}{d \mu}=Z_{\theta}(1), \text { with } \quad Z_{\theta}(t):=\exp \left\{\int_{0}^{t} \theta^{\prime}(s) d W(s)-\frac{1}{2} \int_{0}^{t}\|\theta(s)\|^{2} d s\right\}
$$

for $0 \leq t \leq 1, \theta(\cdot) \in \Theta$. From the Girsanov theorem (e.g. Karatzas \& Shreve (1991), p. 191) we know that under $\mu_{\theta}$, the process $W_{\theta}(\cdot):=W(\cdot)-\int_{0}^{\cdot} \theta(s) d s$ is Brownian motion (or equivalently, that $W(\cdot)$ is Brownian motion with random drift $\theta(\cdot)$ ).

Example 5.1 Simple Hypothesis $\mathbf{Q}=\{\mu\}$ vs. Composite Alternative $\mathbf{P}=\left\{\mu_{\theta}, \theta \in \Theta\right\}$. In this case $H=1, G_{\theta}:=d \mu_{\theta} / d \mu=Z_{\theta}(1)$, and the set $\mathcal{G}:=\left\{G_{\theta}\right\}_{\theta \in \Theta}=\left\{Z_{\theta}(1)\right\}_{\theta \in \Theta}$ is convex, bounded in $\mathbf{L}^{p}(\mu)$ for every $p>1$, and closed under $\mu$-a.e. convergence (see Beneš (1971), pp. 463 and 469). Suppose that

$$
\inf _{\vartheta \in K}\|\vartheta\|=\|\hat{\vartheta}\|, \quad \text { for some } \hat{\vartheta} \in K .
$$

Then it can be shown (cf. Section 6 for details) that the value function of the auxiliary dual problem in (3.11) is given by

$$
\begin{aligned}
\tilde{V}(z) & =\inf _{\theta \in \Theta} \mathbf{E}^{\mu}\left(Z_{\theta}(1)-z\right)^{+}=\mathbf{E}^{\mu}\left(Z_{\hat{\vartheta}}(1)-z\right)^{+} \\
& =\int_{\Re^{d}}\left(e^{\hat{\vartheta} \xi-\frac{1}{2}\|\hat{\vartheta}\|^{2}}-z\right)^{+} \frac{e^{-\frac{1}{2}\|\xi\|^{2}}}{(2 \pi)^{d / 2}} d \xi .
\end{aligned}
$$

Denoting by $\hat{z}$ the unique number in $(0, \infty)$ where $z \mapsto \alpha z+\tilde{V}(z)$ attains its minimum, we obtain the optimal test of Theorem 4.1 in the (pure, non-randomized) form

$$
\hat{X}=\mathbf{1}_{\left\{G_{\hat{\vartheta}}>\hat{z}\right\}}=\mathbf{1}_{\left\{\hat{\vartheta}^{\prime} W(1)>\frac{1}{2}\|\hat{\vartheta}\|^{2}+\log \hat{z}\right\}} .
$$

This test rejects the hypothesis $Q=\mu$, if the inner product of $W(1)$ with the vector $\hat{\vartheta} \in K$ is sufficiently large. With $\Phi(x) \equiv(2 \pi)^{-1 / 2} \int_{-\infty}^{x} e^{-u^{2} / 2} d u$, we have then

$$
\alpha=\mathbf{E}^{\mu}(\hat{X})=\mu\left[\hat{\vartheta}^{\prime} W(1)>\|\hat{\vartheta}\|^{2} / 2+\log \hat{z}\right]=1-\Phi\left(\frac{\log \hat{z}+\frac{1}{2}\|\hat{\vartheta}\|^{2}}{\|\hat{\vartheta}\|}\right)
$$


and thus

$$
\log \frac{1}{\hat{z}}=\frac{1}{2}\|\hat{\vartheta}\|^{2}+\|\hat{\vartheta}\| \cdot \Phi^{-1}(\alpha)
$$

Example 5.2 Composite Hypothesis $\mathbf{Q}=\left\{\mu_{\theta}, \theta \in \Theta\right\}$ vs. Simple Alternative $\mathbf{P}=\{\mu\}$. Here $G=1$ and $H_{\theta}:=d \mu_{\theta} / d \mu=Z_{\theta}(1)$, so that the set $\left\{H_{\theta}\right\}_{\theta \in \Theta}$ is convex and closed under $\mu$-a.e. convergence (cf. Remark 4.1). It is shown in Section 6 that the auxiliary dual problem of (3.11) has value function

$$
\begin{aligned}
\tilde{V}(z) & =\inf _{\theta \in \Theta} \mathbf{E}^{\mu}\left(1-z Z_{\theta}(1)\right)^{+}=\inf _{\theta \in \Theta} \mathbf{E}^{\mu}\left(1-z \cdot e^{\int_{0}^{1} \theta^{\prime}(s) d W(s)-\frac{1}{2} \int_{0}^{1}\|\theta(s)\|^{2} d s}\right)^{+} \\
& =\mathbf{E}^{\mu}\left(1-z Z_{\hat{\vartheta}}(1)\right)^{+}=\mathbf{E}^{\mu}\left(1-z \cdot e^{\hat{\vartheta}^{\prime} W(1)-\frac{1}{2}\|\hat{\vartheta}\|^{2}}\right)^{+} \\
& =\int_{\Re^{d}}\left(1-z \cdot e^{\hat{\vartheta}^{\prime} \xi-\frac{1}{2}\|\hat{\vartheta}\|^{2}}\right)^{+} \frac{e^{-\frac{1}{2}\|\xi\|^{2}}}{(2 \pi)^{d / 2}} d \xi
\end{aligned}
$$

under the assumption (5.2). With $\hat{z}=\arg \min _{0<z<\infty}[\alpha z+\tilde{V}(z)]$ the number of Lemma 4.3, we have now the optimal test of Theorem 4.1 in the (pure, non-randomized) form

$$
\hat{X}=\mathbf{1}_{\left\{\hat{z} H_{\hat{\vartheta}}<1\right\}}=\mathbf{1}_{\left\{\hat{\vartheta}^{\prime} W(1)<\frac{1}{2}\|\hat{\vartheta}\|^{2}-\log \hat{z}\right\}} \cdot
$$

This test rejects the hypothesis, if the inner product of $W(1)$ with the vector $\hat{\vartheta}$ of $(5.2)$ is sufficiently small. In particular, we have

$$
\begin{aligned}
\alpha & =\sup _{\theta \in \Theta} \mathbf{E}^{\mu_{\theta}}(\hat{X})=\sup _{\theta \in \Theta} \mu_{\theta}\left[\hat{\vartheta}^{\prime} W(1)<\|\hat{\vartheta}\|^{2} / 2-\log \hat{z}\right] \\
& =\sup _{\theta \in \Theta} \mu_{\theta}\left[\hat{\vartheta}^{\prime}\left(W_{\theta}(1)+\int_{0}^{1} \theta(s) d s\right)<\|\hat{\vartheta}\|^{2} / 2-\log \hat{z}\right] \\
& =\mu_{\hat{\vartheta}}\left[\hat{\vartheta}^{\prime}\left(W_{\hat{\vartheta}}(1)+\hat{\vartheta}\right)<\|\hat{\vartheta}\|^{2} / 2-\log \hat{z}\right] \\
& =\mu\left[\hat{\vartheta}^{\prime} W(1)<-\|\hat{\vartheta}\|^{2} / 2-\log \hat{z}\right]=\Phi\left(-\frac{\log \hat{z}+\|\hat{\vartheta}\|^{2} / 2}{\|\hat{\vartheta}\|}\right)
\end{aligned}
$$

provided that the vector $\hat{\vartheta} \in K$ of (5.2) satisfies

$$
\hat{\vartheta}^{\prime}(\vartheta-\hat{\vartheta}) \geq 0, \quad \forall \vartheta \in K
$$

In this case, $\hat{z}$ is given again by

$$
\log \frac{1}{\hat{z}}=\frac{1}{2}\|\hat{\vartheta}\|^{2}+\|\hat{\vartheta}\| \cdot \Phi^{-1}(\alpha) .
$$

Example 5.3 Composite Hypothesis $\mathbf{Q}=\left\{\mu_{\theta}, \theta \in \Theta_{1}\right\}$ vs. Composite Alternative $\mathbf{P}=$ $\left\{\mu_{\theta}, \theta \in \Theta_{2}\right\}$. Here $\Theta_{i}$ is the space of $\mathbf{F}$-progressively measurable processes $\theta:[0,1] \times \Omega \rightarrow$ 
$K_{i}, i=1,2$, where $K_{1}$ and $K_{2}$ are closed, convex subsets of $\Re^{d}$ with $K_{1} \cap K_{2}=\emptyset$. We shall assume that there exist vectors $\hat{\vartheta}_{1} \in K_{1}, \hat{\vartheta}_{2} \in K_{2}$ such that

$$
\begin{gathered}
\inf _{\substack{\vartheta_{1} \in K_{1} \\
\vartheta_{2} \in K_{2}}}\left\|\vartheta_{2}-\vartheta_{1}\right\|=\left\|\hat{\vartheta}_{2}-\hat{\vartheta}_{1}\right\| ; \\
\left(\hat{\vartheta}_{2}-\hat{\vartheta}_{1}\right)^{\prime}\left(\vartheta_{1}-\hat{\vartheta}_{1}\right) \leq 0, \quad \forall \vartheta_{1} \in K_{1} .
\end{gathered}
$$

In this case $H_{\theta_{1}}=Z_{\theta_{1}}(1)$ for $\theta_{1}(\cdot) \in \Theta_{1}$, and $G_{\theta_{2}}=Z_{\theta_{2}}(1)$ for $\theta_{2}(\cdot) \in \Theta_{2}$; both sets $\left\{H_{\theta}\right\}_{\theta \in \Theta_{1}}$, $\left\{G_{\theta}\right\}_{\theta \in \Theta_{2}}$ are convex and closed under $\mu$-a.e. convergence, so the auxiliary dual problem of (3.11) has value function

$$
\begin{aligned}
\tilde{V}(z) & =\inf _{\substack{\theta_{1}(\cdot) \cdot \in \Theta_{1} \\
\theta_{2}(\cdot) \in \Theta_{2}}} \mathbf{E}^{\mu}\left(Z_{\theta_{2}}(1)-z Z_{\theta_{1}}(1)\right)^{+}=\inf _{\substack{\theta_{1}(\cdot) \cdot(\cdot) \in \Theta_{1} \\
\theta_{2}(\cdot) \in \Theta_{2}}} \mathbf{E}^{\mu_{\theta_{1}}}\left(\frac{Z_{\theta_{2}}(1)}{Z_{\theta_{1}}(1)}-z\right)^{+} \\
& =\inf _{\substack{\theta_{1}(\cdot) \cdot\left(\cdot \Theta_{1} \\
\theta_{2}(\cdot) \in \Theta_{2}\right.}} \mathbf{E}^{\mu_{\theta_{1}}}\left(e^{\int_{0}^{1}\left(\theta_{2}(s)-\theta_{1}(s)\right)^{\prime} d W_{\theta_{1}}(s)-\frac{1}{2} \int_{0}^{1}\left\|\theta_{2}(s)-\theta_{1}(s)\right\|^{2} d s}-z\right)^{+} \\
& =\mathbf{E}^{\mu_{\hat{\vartheta}_{1}}}\left(e^{\left(\hat{\vartheta}_{2}-\hat{\vartheta}_{1}\right)^{\prime} W_{\hat{\vartheta}_{1}}(1)-\frac{1}{2}\left\|\hat{\vartheta}_{2}-\hat{\vartheta}_{1}\right\|^{2}}-z\right)^{+} \\
& =\int_{\Re^{d}}\left(e^{\left(\hat{\vartheta}_{2}-\hat{\vartheta}_{1}\right)^{\prime} \xi-\frac{1}{2}\left\|\hat{\vartheta}_{2}-\hat{\vartheta}_{1}\right\|^{2}}-z\right)^{+} \frac{e^{-\frac{1}{2}\|\xi\|^{2}}}{(2 \pi)^{d / 2}} d \xi
\end{aligned}
$$

by analogy with (5.3), thanks to the first assumption of (5.10). Again, if we denote by $\hat{z}$ the unique $\arg \min _{0<z<\infty}[\alpha z+\tilde{V}(z)]$, the max-min optimal test of Theorem 4.1 has the form

$$
\hat{X}=1_{\left\{Z_{\hat{\vartheta}_{2}}(1)>\hat{z} Z_{\hat{\vartheta}_{1}}(1)\right\}}=\mathbf{1}_{\left\{\left(\hat{\vartheta}_{2}-\hat{\vartheta}_{1}\right)^{\prime} W(1)>\frac{1}{2}\left(\left\|\hat{\vartheta}_{2}\right\|^{2}-\left\|\hat{\vartheta}_{1}\right\|^{2}\right)+\log \hat{z}\right\}} .
$$

This test rejects the hypothesis, if the inner product of $W(1)$ with $\hat{\vartheta}_{2}$ is sufficiently larger than its inner product with $\hat{\vartheta}_{1}$. Under the second assumption of (5.10), we also have

$$
\begin{aligned}
\alpha & =\sup _{\theta_{1}(\cdot) \in \Theta_{1}} \mathbf{E}^{\mu_{\theta_{1}}}(\hat{X})=\sup _{\theta_{1}(\cdot) \in \Theta_{1}} \mu_{\theta_{1}}\left[\left(\hat{\vartheta}_{2}-\hat{\vartheta}_{1}\right)^{\prime} W(1)>\left(\left\|\hat{\vartheta}_{2}\right\|^{2}-\left\|\hat{\vartheta}_{1}\right\|^{2}\right) / 2+\log \hat{z}\right] \\
& =\sup _{\theta_{1}(\cdot) \in \Theta_{1}} \mu_{\theta_{1}}\left[\left(\hat{\vartheta}_{2}-\hat{\vartheta}_{1}\right)^{\prime} W_{\theta_{1}}(1)+\left(\hat{\vartheta}_{2}-\hat{\vartheta}_{1}\right)^{\prime} \int_{0}^{1} \theta_{1}(s) d s>\frac{1}{2}\left(\left\|\hat{\vartheta}_{2}\right\|^{2}-\left\|\hat{\vartheta}_{1}\right\|^{2}\right)+\log \hat{z}\right] \\
& =\mu_{\hat{\vartheta}_{1}}\left[\left(\hat{\vartheta}_{2}-\hat{\vartheta}_{1}\right)^{\prime} W_{\hat{\vartheta}_{1}}(1)+\left(\hat{\vartheta}_{2}-\hat{\vartheta}_{1}\right)^{\prime} \hat{\vartheta}_{1}>\left(\left\|\hat{\vartheta}_{2}\right\|^{2}-\left\|\hat{\vartheta}_{1}\right\|^{2}\right) / 2+\log \hat{z}\right] \\
& =\mu_{\hat{\vartheta}_{1}}\left[\left(\hat{\vartheta}_{2}-\hat{\vartheta}_{1}\right)^{\prime} W_{\hat{\vartheta}_{1}}(1)>\left\|\hat{\vartheta}_{2}-\hat{\vartheta}_{1}\right\|^{2} / 2+\log \hat{z}\right]
\end{aligned}
$$

from which we deduce

$$
\log \frac{1}{\hat{z}}=\frac{1}{2}\left\|\hat{\vartheta}_{1}-\hat{\vartheta}_{2}\right\|^{2}+\left\|\hat{\vartheta}_{1}-\hat{\vartheta}_{2}\right\| \cdot \Phi^{-1}(\alpha) .
$$


For our next few examples, let us switch to a new setting with Gaussian probability measures

$$
\nu(d \xi)=(2 \pi)^{-d / 2} e^{-\frac{1}{2}\|\xi\|^{2}} d \xi, \quad \nu_{\vartheta}(d \xi)=(2 \pi)^{-d / 2} e^{-\frac{1}{2}\|\xi-\vartheta\|^{2}} d \xi
$$

on $\left(\Re^{d}, \mathcal{B}\left(\Re^{d}\right)\right)$; here again $K$ is a compact, convex subset of $\Re^{d}$ with $0 \notin K$ and such that (5.2) holds. We shall denote by $W$ the identity mapping on $\Re^{d}$.

Example 5.4 Composite Hypothesis $\mathbf{Q}=\left\{\nu_{\vartheta}, \vartheta \in K\right\}$ vs. Simple Alternative $\mathbf{P}=\{\nu\}$. In this new setting, we have $G=1, H_{\vartheta}=d \nu_{\vartheta} / d \nu=e^{\vartheta^{\prime} W-\frac{1}{2}\|\vartheta\|^{2}}$ for every $\vartheta \in K$. From Remark 4.1 , the auxiliary dual problem of (3.11) has now value function

$$
\begin{aligned}
\tilde{V}(z) & =\inf _{\substack{\vartheta_{1}, \vartheta_{2} \in K \\
0 \leq \lambda \leq 1}} \mathbf{E}^{\nu}\left(1-z\left[\lambda H_{\vartheta_{1}}+(1-\lambda) H_{\vartheta_{2}}\right]\right)^{+} \\
& =\inf _{\substack{\vartheta_{1}, \vartheta_{2} \in K \\
0 \leq \lambda \leq 1}} \mathbf{E}^{\nu}\left(1-z\left[\lambda e^{\vartheta_{1}^{\prime} W-\frac{1}{2}\left\|\vartheta_{1}\right\|^{2}}+(1-\lambda) e^{\vartheta_{2}^{\prime} W-\frac{1}{2}\left\|\vartheta_{2}\right\|^{2}}\right]\right)^{+} \\
& \geq \inf _{\theta(\cdot) \in \Theta} \mathbf{E}^{\mu}\left(1-z \cdot e^{\int_{0}^{1} \theta^{\prime}(s) d W(s)-\frac{1}{2} \int_{0}^{1}\|\theta(s)\|^{2} d s}\right)^{+} \\
& =\mathbf{E}^{\mu}\left(1-z \cdot e^{\hat{\vartheta}^{\prime} W(1)-\frac{1}{2}\|\hat{\vartheta}\|^{2}}\right)^{+}=\mathbf{E}^{\nu}\left(1-z \cdot e^{\hat{\vartheta}^{\prime} W-\frac{1}{2}\|\hat{\vartheta}\|^{2}}\right)^{+}
\end{aligned}
$$

thanks to Example 5.2. Thus, the first infimum in (5.15) is attained by, say, $\vartheta_{1}=\hat{\vartheta}, \vartheta_{2} \in K$ arbitrary, $\lambda=1$, and we have

$$
\tilde{V}(z)=\mathbf{E}^{\nu}\left(1-z \cdot e^{\hat{\vartheta}^{\prime} W-\frac{1}{2}\|\hat{\vartheta}\|^{2}}\right)^{+}=\int_{\Re^{d}}\left(1-z \cdot e^{\hat{\vartheta}^{\prime} \xi-\frac{1}{2}\|\hat{\vartheta}\|^{2}}\right)^{+} \frac{e^{-\frac{1}{2}\|\xi\|^{2}}}{(2 \pi)^{d / 2}} d \xi
$$

as in (5.6). The test

$$
\hat{X}=\mathbf{1}_{\left\{\hat{z} H_{\hat{\vartheta}}<1\right\}}=\mathbf{1}_{\left\{\hat{\vartheta}^{\prime} W<\frac{1}{2}\|\hat{\vartheta}\|^{2}-\log \hat{z}\right\}}
$$

of (5.7) rejects the hypothesis if the inner product of $W$ with the vector $\hat{\vartheta} \in K$ of (5.2) is sufficiently small. Here $\hat{z}$ is given once again by (5.5).

Example 5.5 Composite Hypothesis $\mathbf{Q}=\left\{\nu_{\vartheta}, \vartheta \in K_{1}\right\}$ vs. Composite Alternative $\mathbf{P}=$ $\left\{\nu_{\vartheta}, \vartheta \in K_{2}\right\}$, where $K_{1}, K_{2}$ are two disjoint, closed and convex subsets of $\Re^{d}$ that satisfy the conditions of (5.10) for some $\hat{\vartheta}_{1} \in K_{1}, \hat{\vartheta}_{2} \in K_{2}$. (For instance, these conditions hold if $d=1$ and if $K_{1}, K_{2}$ are two disjoint, closed intervals of the real line.)

It is easy to see from the analysis of Example 5.3, that the test

$$
\hat{X}=\mathbf{1}_{\left\{\left(\hat{\vartheta}_{2}-\hat{\vartheta}_{1}\right) W>\frac{1}{2}\left(\left\|\hat{\vartheta}_{2}\right\|^{2}-\left\|\hat{\vartheta}_{1}\right\|^{2}\right)+\log \hat{z}\right\}}
$$

is max-min optimal, where

$$
\hat{z}=\exp \left\{-\left\|\hat{\vartheta}_{1}-\hat{\vartheta}_{2}\right\|^{2} / 2-\Phi^{-1}(\alpha) \cdot\left\|\hat{\vartheta}_{1}-\hat{\vartheta}_{2}\right\|\right\}
$$


attains the infimum of $z \mapsto \alpha z+\tilde{V}(z)$, and

$$
\tilde{V}(z)=\int_{\Re^{d}}\left(e^{\left(\hat{\vartheta}_{2}-\hat{\vartheta}_{1}\right)^{\prime} \xi-\frac{1}{2}\left\|\hat{\vartheta}_{2}-\hat{\vartheta}_{1}\right\|^{2}}-z\right) \frac{e^{-\frac{1}{2}\|\xi\|^{2}}}{(2 \pi)^{d / 2}} d \xi
$$

is the value function of the auxiliary dual problem (3.11).

Example 5.6 Composite Hypothesis $\mathbf{Q}=\left\{\nu_{\vartheta}, \nu_{-\vartheta}\right\}$ vs. Simple Alternative $\mathbf{P}=\{\nu\}$, for some given $\vartheta \in \Re^{d} \backslash\{0\}$. From Remark 4.1, the value function is now

$$
\begin{aligned}
\tilde{V}(z) & =\inf _{0 \leq \lambda \leq 1} \mathbf{E}^{\nu}\left(1-z\left[\lambda e^{\vartheta^{\prime} W-\frac{1}{2}\|\vartheta\|^{2}}+(1-\lambda) e^{-\vartheta^{\prime} W-\frac{1}{2}\|\vartheta\|^{2}}\right]\right)^{+} \\
& =\inf _{|\delta| \leq \frac{1}{2}} \mathbf{E}^{\nu}\left(1-z e^{-\frac{1}{2}\|\vartheta\|^{2}}\left[\left(\frac{1}{2}-\delta\right) e^{\vartheta^{\prime} W}+\left(\frac{1}{2}+\delta\right) e^{-\vartheta^{\prime} W}\right]\right)^{+}=\inf _{|\delta| \leq \frac{1}{2}} f(z ; \delta),
\end{aligned}
$$

where

$$
f(z ; \delta):=\mathbf{E}^{\nu}\left(1+z e^{-\frac{1}{2}\|\vartheta\|^{2}}\left[2 \delta \sinh \left(\vartheta^{\prime} W\right)-\cosh \left(\vartheta^{\prime} W\right)\right]\right)^{+} .
$$

For every fixed $z \in(0, \infty)$, the function $\delta \mapsto f(z ; \delta)$ is symmetric and attains its infimum over $\left[-\frac{1}{2}, \frac{1}{2}\right]$ at $\delta=0$, so that

$$
\tilde{V}(z)=f(z ; 0)=\mathbf{E}^{\nu}\left(1-z e^{-\frac{1}{2}\|\vartheta\|^{2}} \cosh \left(\vartheta^{\prime} W\right)\right)^{+} .
$$

The optimal max-min test of (5.7), namely

$$
\hat{X}=\mathbf{1}_{\left\{\hat{z}\left(e^{\vartheta^{\prime} W-\frac{1}{2}\|\vartheta\|^{2}}+e^{-\vartheta^{\prime} W-\frac{1}{2}\|\vartheta\|^{2}}\right)<2\right\}}=\mathbf{1}_{\left\{\hat{z} e^{-\frac{1}{2}\|\vartheta\|^{2}} \cosh \left(\vartheta^{\prime} W\right)<1\right\}}=\mathbf{1}_{\left\{\left|\vartheta^{\prime} W\right|<h\|\vartheta\|\right\}},
$$

rejects the hypothesis if the absolute value of the inner product $\vartheta^{\prime} W$ is sufficiently small. Here we have set

$$
h:=\frac{1}{\|\vartheta\|} \cosh ^{-1}\left(\frac{e^{\frac{1}{2}\|\vartheta\|^{2}}}{\hat{z}}\right),
$$

where $\cosh ^{-1}(\cdot)$ is the inverse of $\cosh (\cdot)$ on $(0, \infty)$ with $\cosh ^{-1}(u) \equiv 0$ for $0 \leq u \leq 1$, and $\hat{z}=\arg \min _{0<z<\infty}[\alpha z+\tilde{V}(z)]$. The quantity $h>0$ of (5.18) is characterized by

$$
\alpha=\nu_{ \pm \theta}\left(\left|\vartheta^{\prime} W\right|<h\|\vartheta\|\right)=\nu\left(\left|\vartheta^{\prime}(W \pm \vartheta)\right|<h\|\vartheta\|\right)=\nu(|\Xi \pm\|\vartheta\||<h)
$$

where $\Xi:=\vartheta^{\prime} W /\|\vartheta\|$ is standard normal under $\nu$, or equivalently by the equation

$$
\Phi(\|\vartheta\|+h)-\Phi(\|\vartheta\|-h)=\alpha
$$

Remark 5.1 It is not hard to verify that the test of (5.17)-(5.19) is also max-min optimal for testing the composite hypothesis $\mathbf{Q}=\left\{\nu_{m}, m \in(-\infty,-\vartheta] \cup[\vartheta, \infty)\right\}$ vs. the simple alternative $\mathbf{P}=\{\nu\}$, for some $\vartheta>0$, in the case $d=1$. 


\section{Proofs}

Proof of (3.2): The convexity of the set (3.1) is obvious; now if $\left\{H_{n}\right\}_{n \in \mathbf{N}} \subseteq \mathcal{H}$ and $\lim _{n} H_{n}=H, \mu$-a.e., then clearly $H \geq 0, \mu$-a.e. and

$$
\mathbf{E}^{\mu}(H X)=\mathbf{E}\left[\lim _{n}\left(H_{n} X\right)\right] \leq \liminf _{n} \mathbf{E}\left(H_{n} X\right) \leq \alpha
$$

for every $X \in \mathcal{X}_{\alpha}$ by Fatou's lemma, so $H \in \mathcal{H}$. In other words, $\mathcal{H}$ is closed under $\mu$-a.e. convergence. On the other hand, every $H$ of the form $H_{Q}=d Q / d \mu$ for some $Q \in \mathbf{Q}$, belongs to $\mathcal{H}$, so that (3.2) holds as well, since $\mathbf{E}^{\mu}\left(H_{Q} X\right)=\mathbf{E}^{Q}(X) \leq \alpha, \forall X \in \mathcal{X}_{\alpha}$ from (2.4). Finally, from (2.4) the degenerate random variable $X \equiv \alpha$ belongs to $\mathcal{X}_{\alpha}$; with this choice in (3.1) we see that $\mathbf{E}^{\mu}(H) \leq 1, \forall H \in \mathcal{H}$, so that $\mathcal{H}$ is a bounded subset of $\mathbf{L}^{1}(\mu)$.

Proof of Proposition 3.1: From (3.4), (3.5) and (2.8) we have

$$
\begin{gathered}
\mathbf{E}^{\hat{P}}(X) \leq \alpha \hat{z}+\mathbf{E}^{\mu}(\hat{G}-\hat{z} \hat{H})^{+}, \quad \forall X \in \mathcal{X}_{\alpha}, \\
\left.\mathbf{E}^{\hat{P}}(\hat{X})=\hat{z} \cdot \mathbf{E}^{\mu}(\hat{H} \hat{X})+\mathbf{E}^{\mu}(\hat{G}-\hat{z} \hat{H}) \hat{X}\right]=\alpha \hat{z}+\mathbf{E}^{\mu}(\hat{G}-\hat{z} \hat{H})^{+} .
\end{gathered}
$$

This leads to the first inequality in (3.8). On the other hand, (3.4) and (3.5) also give

$$
\begin{aligned}
\mathbf{E}^{P}(\hat{X}) & =\hat{z} \cdot \mathbf{E}^{\mu}(\hat{H} \hat{X})+\mathbf{E}^{\mu}\left[\hat{X}\left(G_{P}-\hat{z} \hat{H}\right)\right] \\
& =\alpha \hat{z}+\mathbf{E}^{\mu}\left[\hat{X}\left(G_{P}-\hat{z} \hat{H}\right)\right], \quad \forall P \in \mathbf{P} .
\end{aligned}
$$

Thanks to the assumption (3.7), this last quantity dominates

$$
\alpha \hat{z}+\mathbf{E}^{\mu}[\hat{X}(\hat{G}-\hat{z} \hat{H})]=\mathbf{E}^{\hat{P}}(\hat{X})
$$

and the second inequality of (3.8) follows.

Proof of Proposition 3.2: From (3.4) we have

$$
\mathbf{E}^{\mu}(G-z H)^{+}+\alpha z \geq \mathbf{E}^{P}(X), \quad \forall(G, H) \in \mathcal{G} \times \mathcal{H}
$$

for every $z>0, X \in \mathcal{X}_{\alpha}$ and with $P=\int G d \mu$. On the other hand, (3.6) gives

$$
\mathbf{E}^{\mu}(\hat{G}-\hat{z} \hat{H})^{+}+\alpha \hat{z}=\mathbf{E}^{\hat{P}}(\hat{X}) .
$$

Now read (6.1) with $z=\hat{z}, X=\hat{X}$ to obtain (i), in conjunction with $(3.6)^{\prime}$ and the second inequality of (3.8). Similarly, read (6.1) with $z>0$ arbitrary, and $X \in \mathcal{X}_{\alpha}$, to obtain (ii) and $V_{*}(\alpha)=\mathbf{E}^{\hat{P}}(\hat{X})$, again in conjunction with $(3.6)^{\prime}$ and the second inequality of (3.8). The property (iv) follows then from Proposition 3.1, and (iii) is an easy consequence of (i), (ii). 
Proof of Lemma 4.1: From $\left(G-z_{1} H\right)^{+}-\left(G-z_{2} H\right)^{+} \leq\left|z_{1}-z_{2}\right| H$, from $\mathbf{E}^{\mu}(H) \leq 1$, and from (3.11), we get

$$
\begin{aligned}
\tilde{V}\left(z_{1}\right) & \leq \mathbf{E}^{\mu}\left(G-z_{1} H\right)^{+} \leq\left|z_{1}-z_{2}\right| \cdot \mathbf{E}^{\mu}(H)+\mathbf{E}^{\mu}\left(G-z_{2} H\right)^{+} \\
& \leq \mathbf{E}^{\mu}\left(G-z_{2} H\right)^{+}+\left|z_{1}-z_{2}\right|, \quad \forall(G, H) \in \mathcal{G} \times \mathcal{H} .
\end{aligned}
$$

Taking infimum over this set, we obtain $\tilde{V}\left(z_{1}\right) \leq \tilde{V}\left(z_{2}\right)+\left|z_{1}-z_{2}\right|$, and then we just interchange the roles of $z_{1}$ and $z_{2}$.

Proof of Lemma 4.2: Let $\left\{\left(G_{n}, H_{n}\right)\right\}_{n \in \mathbf{N}} \subset \mathcal{G} \times \mathcal{H}$ be a minimizing sequence for (3.11). Because $\mathcal{G} \times \mathcal{H}$ is bounded in $\mathbf{L}^{1}(\mu) \times \mathbf{L}^{1}(\mu)$, there exists by the theorem of Komlós (1967) (see also Schwartz (1986)) a random vector $(\hat{G}, \hat{H}) \in \mathbf{L}^{1}(\mu) \times \mathbf{L}^{1}(\mu)$ and a relabelled subsequence $\left\{\left(G_{j}^{\prime}, H_{j}^{\prime}\right)\right\}_{j \in \mathbf{N}} \subseteq\left\{\left(G_{n}, H_{n}\right)\right\}_{n \in \mathbf{N}}$, such that

$$
\left(\tilde{G}_{k}, \tilde{H}_{k}\right):=\left(\frac{1}{k} \sum_{j=1}^{k} G_{j}^{\prime}, \frac{1}{k} \sum_{j=1}^{k} H_{j}^{\prime}\right) \longrightarrow(\hat{G}, \hat{H})
$$

as $k \rightarrow \infty, \mu$-a.e. By the convexity and the $\mu$-a.e. closedness of $\mathcal{G}$ and $\mathcal{H}$, we have $(\hat{G}, \hat{H}) \in \mathcal{G} \times \mathcal{H}$; on the other hand, Fatou's lemma and convexity give

$$
\begin{aligned}
\mathbf{E}^{\mu}(\hat{G}-z \hat{H})^{+} & =\mathbf{E}^{\mu}\left[\lim _{k}\left(\tilde{G}_{k}-z \tilde{H}_{k}\right)^{+}\right] \\
& \leq \liminf _{k} \mathbf{E}^{\mu}\left(\tilde{G}_{k}-z \tilde{H}_{k}\right)^{+}=\liminf _{k} \mathbf{E}^{\mu}\left(\frac{1}{k} \sum_{j=1}^{k}\left(G_{j}^{\prime}-z H_{j}^{\prime}\right)\right)^{+} \\
& \leq \liminf _{k} \mathbf{E}^{\mu}\left(\frac{1}{k} \sum_{j=1}^{k}\left(G_{j}^{\prime}-z H_{j}^{\prime}\right)^{+}\right)=\lim _{j} \mathbf{E}^{\mu}\left(G_{j}^{\prime}-z H_{j}^{\prime}\right)^{+}=\tilde{V}(z) .
\end{aligned}
$$

Proof of Lemma 4.3: The convex function

$$
f_{\alpha}(z):=\alpha z+\tilde{V}(z)=\alpha z+\inf _{(G, H) \in(\mathcal{G} \times \mathcal{H})} \mathbf{E}^{\mu}(G-z H)^{+}, \quad 0<z<\infty
$$

satisfies $f_{\alpha}(0+)=\inf _{G \in \mathcal{G}} \mathbf{E}^{\mu}(G)=1, f_{\alpha}(z) \geq \alpha z$ and thus $f_{\alpha}(\infty)=\infty$. Consequently, $f_{\alpha}(\cdot)$ either attains its infimum at some $\hat{z}_{\alpha} \in(0, \infty)$ as claimed, or else satisfies $f_{\alpha}(z) \geq 1, \forall z>0$. This latter possibility can be ruled out easily, as it implies

$\alpha z \geq 1-\tilde{V}(z) \geq 1-\mathbf{E}^{\mu}(G-z H)^{+}=\mathbf{E}^{\mu}(G)-\mathbf{E}^{\mu}(G-z H)^{+} \geq z \cdot \mathbf{E}^{\mu}\left[H \mathbf{1}_{\{z H \leq G\}}\right], \quad \forall z>0$

for any given $G \in \mathcal{G}, H \in \mathcal{H}$; dividing by $z$ and then letting $z \downarrow 0$, we obtain $\mathbf{E}^{\mu}(H) \leq \alpha$ for every $H \in \mathcal{H}$, and thus $\alpha \geq 1$, a contradiction.

Proof of Lemma 4.4: The convexity of $\mathcal{G}$ and $\mathcal{H}$ leads to that of the set $\mathcal{M}$ in (4.2). Now consider a sequence $\left\{\left(G_{n}, H_{n}, z_{n}\right)\right\}_{n \in \mathbf{N}} \subseteq \mathcal{M}$ which converges to some $(G, H, z) \in \mathcal{L}$ 
in the norm $\|\cdot\|$ of Lemma 4.4. Then $z_{n} \rightarrow z$ and $\left(G_{n_{j}}, H_{n_{j}}\right) \rightarrow(G, H)$, $\mu$-a.e. (along a subsequence) so that $G \in \mathcal{G}$ and $H \in \mathcal{H}$ by the closedness of both $\mathcal{G}$ and $\mathcal{H}$ under $\mu$-a.e. convergence. The properness, convexity and lower-semicontinuity of the functional (4.3) on $\mathcal{L}$ are relatively easy to check. For the final claim of the lemma, just observe from (3.13), (3.11) that we have

$$
\alpha \hat{z}+\mathbf{E}^{\mu}(\hat{G}-\hat{z} \hat{H})^{+}=\alpha \hat{z}+\tilde{V}(\hat{z}) \leq \alpha z+\tilde{V}(z) \leq \alpha z+\mathbf{E}^{\mu}(G-z H)^{+} \quad, \quad \forall(G, H, z) \in \mathcal{M} .
$$

Proof of Theorem 4.1: First, let us read (4.7) with

$$
G:=\hat{G}-\mathbf{1}_{\{\hat{X}+\hat{W}>0\}}, \quad Y:=\hat{z} \hat{H}-\mathbf{1}_{\{\hat{X}+\hat{W}>0\}}
$$

and in conjunction with (4.8), to obtain $\mathbf{E}^{\mu}(\hat{X}+\hat{W})^{+}=\mathbf{E}^{\mu}\left[(\hat{X}+\hat{W}) \mathbf{1}_{\{\hat{X}+\hat{W} \geq 0\}}\right] \leq 0$. Similarly, we can derive $\mathbf{E}^{\mu}(\hat{X}+\hat{W})^{-} \leq 0$, whence $\hat{X}+\hat{W}=0, \mu$-a.e. This proves the first equality in (4.9) and shows, in conjunction with (4.8), that the conditions of (4.6), (4.7) can be written as

$$
\begin{gathered}
\mathbf{E}^{\mu}[\hat{X}(\hat{z} \hat{H}-z H+G-\hat{G})] \geq \alpha(\hat{z}-z), \quad \forall(G, H, z) \in \mathcal{M} \\
\mathbf{E}^{\mu}\left[\hat{X}(Y-\hat{z} \hat{H}+\hat{G}-G)+(G-Y)^{+}-(\hat{G}-\hat{z} \hat{H})^{+}\right] \geq 0, \quad \forall(G, Y) \in\left(\mathbf{L}^{1}(\mu)\right)^{2} .
\end{gathered}
$$

From now on we shall write

$$
\hat{X}=\mathbf{1}_{\{\hat{z} \hat{H}<\hat{G}\}}+A
$$

for a suitable random variable $A$. Reading $(4.7)^{\prime}$ with $G \equiv \hat{G}$ and $\hat{X}$ as in (6.2) we obtain

$$
\begin{array}{r}
\mathbf{E}^{\mu}[(\hat{z} \hat{H}-Y) A] \leq \mathbf{E}^{\mu}\left[(\hat{G}-Y)^{+}-(\hat{G}-\hat{z} \hat{H}) \mathbf{1}_{\{\hat{z} \hat{H}<\hat{G}\}}+(Y-\hat{z} \hat{H}) \mathbf{1}_{\{\hat{z} \hat{H}<\hat{G}\}}\right] \\
=\mathbf{E}^{\mu}\left[(\hat{G}-Y) \mathbf{1}_{\{Y<\hat{G}\}}+(Y-\hat{G}) \mathbf{1}_{\{\hat{z} \hat{H}<\hat{G}\}}\right], \quad \forall \quad Y \in \mathbf{L}^{1}(\mu) .
\end{array}
$$

Now take $Y \in \mathbf{L}^{1}(\mu)$ such that

$$
\{Y<\hat{G}\}=\{\hat{z} \hat{H}<\hat{G}\}, \bmod . \mu
$$

which implies

$$
\mathbf{E}^{\mu}[A(\hat{z} \hat{H}-Y)] \leq 0, \quad \text { for every } \quad Y \in \mathbf{L}^{1}(\mu) \quad \text { as in }(6.3),
$$

as well as

$$
\begin{aligned}
& A \leq 0, \quad \mu-a . e \text {. on }\{\hat{z} \hat{H}<\hat{G}\} \\
& A \geq 0, \quad \mu-a . e . \text { on }\{\hat{z} \hat{H} \geq \hat{G}\} .
\end{aligned}
$$


To prove (6.4), let us assume $\mu(A>0, \hat{z} \hat{H}<\hat{G})>0$; we can select $Y \in \mathbf{L}^{1}(\mu)$ negative and with $|Y|$ so large on this set, as to make $\mathbf{E}^{\mu}\left[A(\hat{z} \hat{H}-Y) \mathbf{1}_{\{A>0, \hat{z} \hat{H}<\hat{G}\}}\right]$ positive and large enough to violate $(4.7)^{\prime \prime \prime}$ written in the form

$$
\mathbf{E}^{\mu}\left[A(\hat{z} \hat{H}-Y) \mathbf{1}_{\{A>0, \hat{z} \hat{H}<\hat{G}\}}\right]+\mathbf{E}^{\mu}\left[A(\hat{z} \hat{H}-Y) \mathbf{1}_{\{A \leq 0, \hat{z} \hat{H}<\hat{G}\}}\right]+\mathbf{E}^{\mu}\left[A(\hat{z} \hat{H}-Y) \mathbf{1}_{\{\hat{z} \hat{H} \geq \hat{G}\}}\right] \leq 0 .
$$

Similarly, in order to prove (6.5), assume that the set $\{A<0, \hat{z} \hat{H} \geq \hat{G}\}$ has positive $\mu$-probability, and select $Y \in \mathbf{L}^{1}(\mu)$ positive and so large on this set, as to violate condition $(4.7)^{\prime \prime \prime}$.

We can also see that

$$
\begin{aligned}
& A=0, \mu-a . e . \text { on }\{\hat{z} \hat{H}<\hat{G}\} \\
& A=0, \quad \mu-a . e . \text { on }\{\hat{z} \hat{H}>\hat{G}\} .
\end{aligned}
$$

To prove (6.6), assume $\mu(A<0, \hat{z} \hat{H}<\hat{G})>0$ so that $\delta:=\mathbf{E}^{\mu}\left[A(\hat{z} \hat{H}-\hat{G}) \mathbf{1}_{\{\hat{z} \hat{H}<\hat{G}\}}\right]>0$ in conjunction with (6.4). For arbitrary $\varepsilon>0$, the random variable

$$
Y:=\left\{\begin{array}{ccc}
\hat{G}-\varepsilon & ; & \text { on }\{\hat{z} \hat{H}<\hat{G}\} \\
\hat{G} & ; & \text { on }\{\hat{z} \hat{H} \geq \hat{G}\}
\end{array}\right\}
$$

is in $\mathbf{L}^{1}(\mu)$, satisfies (6.3), and for it the condition (4.7)"' becomes

$$
\begin{aligned}
0 & \geq \mathbf{E}^{\mu}\left[A(\hat{z} \hat{H}-\hat{G}) \mathbf{1}_{\{\hat{y} \hat{H} \geq \hat{G}\}}\right]+\mathbf{E}^{\mu}\left[A(\hat{z} \hat{H}-\hat{G}+\varepsilon) \mathbf{1}_{\{\hat{z} \hat{H}<\hat{G}\}}\right] \\
& =\mathbf{E}^{\mu}\left[A(\hat{z} \hat{H}-\hat{G}) \mathbf{1}_{\{\hat{z} \hat{H} \geq \hat{G}\}}\right]+\delta+\varepsilon \cdot \mathbf{E}^{\mu}\left[A \mathbf{1}_{\{\hat{z} \hat{H}<\hat{G}\}}\right] .
\end{aligned}
$$

Letting $\varepsilon \downarrow 0$, this gives $\delta+\mathbf{E}^{\mu}\left[A(\hat{z} \hat{H}-\hat{G})^{+}\right] \leq 0$, which is absurd in light of (6.5). This proves the property (6.6), and allows us to recast the condition $(4.7)^{\prime \prime \prime}$ in the equivalent form

$$
\mathbf{E}^{\mu}\left[A(\hat{z} \hat{H}-Y) \mathbf{1}_{\{\hat{z} \hat{H} \geq \hat{G}\}}\right] \leq 0, \quad \text { for every } \quad Y \in \mathbf{L}^{1}(\mu) \text { as in (6.3). }
$$

In particular, with $Y$ as in (6.8), this gives $\mathbf{E}^{\mu}\left[A(\hat{z} \hat{H}-)^{+}\right] \leq 0$ which, in light of (6.5), leads to $(6.7)$.

The properties (6.4)-(6.7) show that we have

$$
A=B \cdot \mathbf{1}_{\{\hat{z} \hat{H}=\hat{G}\}} \text { for some random variable } B \geq 0 .
$$

Back into (4.7)", this gives

$$
\mathbf{E}^{\mu}\left[(\hat{G}-Y) B \mathbf{1}_{\{\hat{z} \hat{H}=\hat{G}\}}\right] \leq \mathbf{E}^{\mu}\left[(\hat{G}-Y) \mathbf{1}_{\{Y<\hat{G}\}}+(Y-\hat{G}) \mathbf{1}_{\{\hat{z} \hat{H}<\hat{G}\}}\right], \forall Y \in \mathbf{L}^{1}(\mu) .
$$

Finally, we have to establish

$$
0 \leq B \leq 1, \quad \mu \text {-a.e. on }\{\hat{z} \hat{H}=\hat{G}\}
$$


In order to show this, we shall read (6.10) with the choice

$$
Y:=\left\{\begin{array}{ccc}
0 & ; & \text { on }\{B>1, \hat{z} \hat{H}=\hat{G}\} \\
\hat{G} & ; & \text { on }\{B \leq 1, \hat{z} \hat{H}=\hat{G}\} \\
\hat{G}(1-\varepsilon) & ; & \text { otherwise }
\end{array}\right\}
$$

for arbitrary $0<\varepsilon<1$; this leads to

$$
\mathbf{E}^{\hat{P}}\left[B \cdot \mathbf{1}_{\{B>1, \hat{z} \hat{H}=\hat{G}\}}\right] \leq \hat{P}[B>1, \hat{z} \hat{H}=\hat{G}]+\varepsilon \cdot \hat{P}[\hat{z} \hat{H}>\hat{G}]
$$

for every $0<\varepsilon<1$, as well as to

$$
\mathbf{E}^{\hat{P}}\left[(B-1)^{+} \cdot \mathbf{1}_{\{\hat{z} \hat{H}=\hat{G}\}}\right] \leq 0
$$

in the limit, as $\varepsilon \downarrow 0$. Therefore, $B \leq 1 \mu$-a.e. on $\{\hat{z} \hat{H}=\hat{G}\}$. This concludes the proof that $\hat{X}$ is of the form (2.8), (4.9). In particular, $\mu(0 \leq \hat{X} \leq 1)=1$ and (3.5)(b) holds, so $\hat{X} \in \mathcal{X}_{\alpha}$.

Proof of (5.3) and (5.6); adapted from Xu \& Shreve (1992): Let $\varphi: \Re \rightarrow[0, \infty)$ be a convex function satisfying a linear growth condition; with $\hat{\vartheta} \in K$ as in (5.2), and arbitrary $\theta(\cdot) \in \Theta$, we shall show

$$
\mathbf{E}^{\mu} \varphi\left(Z_{\theta}(t)\right) \geq \mathbf{E}^{\mu} \varphi\left(Z_{\hat{\vartheta}}(t)\right), \quad 0 \leq t \leq 1
$$

This will clearly prove (5.3) (resp., (5.6)) by taking $t=1$ and $\varphi(x)=(x-z)^{+}$(resp., $\left.\varphi(x)=(1-z x)^{+}\right)$. We can write

$$
\frac{\theta(\cdot)}{\|\theta(\cdot)\|}=Q(\cdot) \frac{\hat{\vartheta}}{\|\hat{\vartheta}\|}, \quad \text { a.e. on }[0,1] \times \Omega
$$

for some $\mathbf{F}$-progressively measurable process $Q(\cdot)$ with values in the space of $(d \times d)$-orthonormal matrices. Let us also notice that the process

$$
\Lambda(t):=\int_{0}^{t} \frac{\|\theta(s)\|^{2}}{\|\hat{\vartheta}\|^{2}} d s \geq t, \quad 0 \leq t \leq 1
$$

is increasing with $\frac{d}{d t} \Lambda(\cdot) \geq 1$, so that its inverse is well defined and $\Lambda^{-1}(u)$ is an $\mathbf{F}$-stopping time with $\Lambda^{-1}(u) \leq u$, for every $0 \leq u \leq 1$. Now the time-changed process

$$
\hat{W}(u):=\int_{0}^{\Lambda^{-1}(u)} \frac{\|\theta(s)\|}{\|\hat{\vartheta}\|} Q^{\prime}(s) d W(s), \quad 0 \leq u \leq 1
$$

is a (local) martingale of the filtration $\hat{\mathcal{F}}(u):=\mathcal{F}\left(\Lambda^{-1}(u)\right), 0 \leq u \leq 1$, with

$$
\left\langle\hat{W}_{i}, \hat{W}_{j}\right\rangle(u)=\delta_{i j} \int_{0}^{\Lambda^{-1}(u)} \frac{\|\theta(s)\|^{2}}{\|\hat{\vartheta}\|^{2}} d s=u \cdot \delta_{i j} .
$$


In other words, $\hat{W}(\cdot)$ is an $\hat{\mathbf{F}}$-Brownian motion (by P. Lévy's Theorem 3.3.16 in Karatzas \& Shreve (1991)), and

$$
\begin{aligned}
1 & +\int_{0}^{u} Z_{\theta}\left(\Lambda^{-1}(\tau)\right) \hat{\vartheta}^{\prime} d \hat{W}(\tau)=1+\int_{0}^{\Lambda^{-1}(u)} Z_{\theta}(s) \hat{\vartheta}^{\prime} d \hat{W}(\Lambda(s)) \\
& =1+\int_{0}^{\Lambda^{-1}(u)} Z_{\theta}(s) \frac{\|\theta(s)\|}{\|\hat{\vartheta}\|}(Q(s) \hat{\vartheta})^{\prime} d W(s)=1+\int_{0}^{\Lambda^{-1}(u)} Z_{\theta}(s) \theta^{\prime}(s) d W(s) \\
& =Z_{\theta}\left(\Lambda^{-1}(u)\right), \quad 0 \leq u \leq 1 .
\end{aligned}
$$

Since $Z_{\hat{\vartheta}}(t)=1+\int_{0}^{t} Z_{\hat{\vartheta}}(s) \hat{\vartheta}^{\prime} d W(s), 0 \leq t \leq 1$, we conclude that the processes $Z_{\hat{\vartheta}}(\cdot)$, $Z_{\theta}\left(\Lambda^{-1}(\cdot)\right)$ have the same distribution. From this, from the optional sampling theorem, and the fact that $\varphi\left(Z_{\theta}(\cdot)\right)$ is a submartingale, we obtain

$$
\mathbf{E}^{\mu} \varphi\left(Z_{\hat{\vartheta}}(t)\right)=\mathbf{E}^{\mu} \varphi\left(Z_{\theta}\left(\Lambda^{-1}(t)\right) \leq \mathbf{E}^{\mu} \varphi\left(Z_{\theta}(t)\right), \quad \text { which proves }(6.10)\right.
$$

Acknowledgement: We are grateful to Professor Hans Föllmer for calling our attention to the book of Witting (1985), and to the referees and the editor for pointing out relevant literature.

\section{$7 \quad$ References}

AUBIN, J. \& EKELAND, I. (1984) Applied Nonlinear Analysis. John Wiley \& Sons, New York.

BAUMANN, V. (1968) Eine papameterfreie Theorie der ungünstigsten Verteilungen für das Testen von Hypothesen. Z. Wahrscheinlichkeitstheorie verw. Gebiete 11, 61-73.

BENEŠ, V.E. (1971) Existence of Optimal Stochastic Control Laws. SIAM Journal of Control 8, 446-475.

CVITANIĆ, J. (2000) Minimizing expected loss of hedging in incomplete and constrained markets. SIAM J. Control \& Optimization, to appear.

CVITANIĆ, J. \& KARATZAS, I. (1999) On dynamic measures of risk. Finance \& Stochastics 3, 451-482.

FERGUSON, T.S. (1967) Mathematical Statistics: A Decision-Theoretic Approach. Academic Press, New York and London.

FÖLLMER, H. \& LEUKERT, P. (1999) Quantile Hedging. Finance \& Stochastics 3, 251273. 
FÖLLMER, H. \& LEUKERT, P. (2000) Partial hedging with minimal shortfall risk. Finance \& Stochastics 4, 117-146.

HEATH, D. (1993) A continuous-time version of Kulldorff's result. Unpublished Manuscript, Cornell University.

HUBER, P. \& STRASSEN, V. (1973) Minimax tests and the Neyman-Pearson lemma for capacities. Ann. Statistics 1, 251-263.

KARATZAS, I. (1997) Adaptive control of a diffusion to a goal, and a parabolic MongeAmpère-type equation. Asian J. Mathematics 1, 295-313.

KARATZAS, I. \& SHREVE, S.E. (1991) Brownian Motion and Stochastic Calculus (2 $2^{\text {nd }}$ edition). Springer-Verlag, New York.

KOMLÓS, J. (1967) A generalization of a problem of Steinhaus. Acta Math. Acad. Sci. Hungar. 18, 217-229.

KRAFFT, 0. \& WITTING, H. (1967) Optimale Tests und ungünstigste Verteilungen. $Z$. Wahrscheinlichkeitstheorie verw. Gebiete 7, 289-302.

LEHMANN, E. L. (1952) On the existence of least-favorable distributions. Ann. Math. Stat. 23, 408-416.

LEHMANN, E. L. (1986) Testing Statistical Hypotheses (2 $2^{\text {nd }}$ edition). John Wiley \& Sons, Inc., New York.

SCHWARTZ, M. (1986) New proofs of a theorem of Komlós. Acta Math. Hung. 47, 181-185. SPIVAK, G. (1998) Maximizing the Probability of Perfect Hedge. Doctoral Dissertation, Department of Statistics, Columbia University.

VAJDA, I. (1989) Theory of Statistical Inference and Information. Kluwer Publishers, Dordrecht.

WITTING, H. (1985) Mathematische Statistik I. B.G. Teubner, Stuttgart.

XU, G.-L. \& SHREVE, S. E. (1992) A duality method for optimal consumption and investment under short-selling prohibition. II: Constant market coefficients. Annals of Applied Probability 2, 314-328. 